\title{
Some results from the Yakutsk array experiment and the status of the modernization program
}

\author{
Anatoly Ivanov ${ }^{1, \star}$ for the Yakutsk array group \\ ${ }^{1}$ Shafer Institute for Cosmophysical Research \& Aeronomy, Yakutsk 677980, Russia
}

\begin{abstract}
The Yakutsk array has been used to study cosmic rays since 1974. Charged particles such as electrons, positrons and muons are detected, as well as radio signals emitted by air showers. However, the characteristic shower component detected by the array is Cherenkov light. This article briefly reviews recent results from the Yakutsk array group.
\end{abstract}

\section{Introduction}

An extensive air shower (EAS) of cosmic rays (CRs) is a cascade of particles induced by an energetic astroparticle hitting the atmosphere. Detection of an EAS is made possible by detectors placed at the Earth's surface and underground, and detectors viewing the atmosphere, operating together. Examples of the detectors used in the Yakutsk array experiment are shown in Fig. 1.

The array can be characterized by the target energy range of the primary CRs detected $\left(10^{15}-\right.$ $10^{19} \mathrm{eV}$ ) and the area over which detectors are located $\left(8 \mathrm{~km}^{2}\right.$ at present, and $17 \mathrm{~km}^{2}$ during the period $1974-$ 1990). The array consists of 58 stations equipped with scintillation counters; 48 Cherenkov light detectors; four underground muon detectors; three detectorsobscura; and six radio detectors $(32 \mathrm{MHz})$.

Shower events are selected based on two-level trigger from the set of detector signals. The first level is the coincidence of two scintillator signals at a station within $2 \mu \mathrm{s}$, while the second level is the coincidence of signals from at least three nearby stations within $40 \mu \mathrm{s}$.

The detection times for the shower front at the surface stations are used to calculate the angles of arrival for the EAS primary particles. A clock pulse transmitter at the center of the array provides pulse timing with an accuracy of $100 \mathrm{~ns}$. The resultant errors in arrival angles depend on the primary energy, which decreases from $\sim 7^{\circ}$ at $E=1 \mathrm{EeV}$ to $\sim 3^{\circ}$ above $E=10 \mathrm{EeV}[1-5]$.

The energy estimation method involves the total flux measurement of the Cherenkov light and the number of electrons and muons at the observation level [6-9]. The error in the energy estimation is the sum of the random and systematic uncertainties in the

^e-mail: ivanov@ikfia.ysn.ru errors in the absolute calibration of the Cherenkov light detectors and in the estimation of the atmospheric transparency. The total error in the energy estimation increases with the zenith angle and the primary energy, and reaches $\delta E / E \sim 35 \%$ for showers with axes within the array area [5].

\section{Recent results involving the interaction of particles and the characteristics of air showers}

A multitude of particles is produced in a shower via inelastic interactions with nuclei in the atmosphere. The main characteristic of the proton-nuclei interaction is the mean total inelastic cross section, and this can be estimated based on a measurement of the attenuation length of the EAS, namely the exponential tail of the distribution of $x_{\max }$, which is related to $\sigma_{p}^{a i r}$ according to model simulations of air showers (Fig. 2, left panel). The Yakutsk array results [10] for the proton-air cross section are shown in the right panel of Fig. 2, and are compared with the results of other experiments and model predictions. In general, the data consistently show a cross section that increases with energy. A detailed analysis of the statistical precision of the experimental results and the systematic uncertainties in the predictions of the model can be found in [11].

The attenuation of showers in the atmosphere used to be studied applying the method of constant intensity cuts [12]. We derived the $S_{600}(x)$ profile and the coupled zenith angle distribution of the EAS event rate (illustrated in Fig. 3) using data from the Yakutsk array [13].

An additional constraint used in data selection is the unified particle density threshold, $\rho_{600}^{t h r}$, which is implemented in place of a multitude of specific detector thresholds. Fluctuations in densities are approx- 


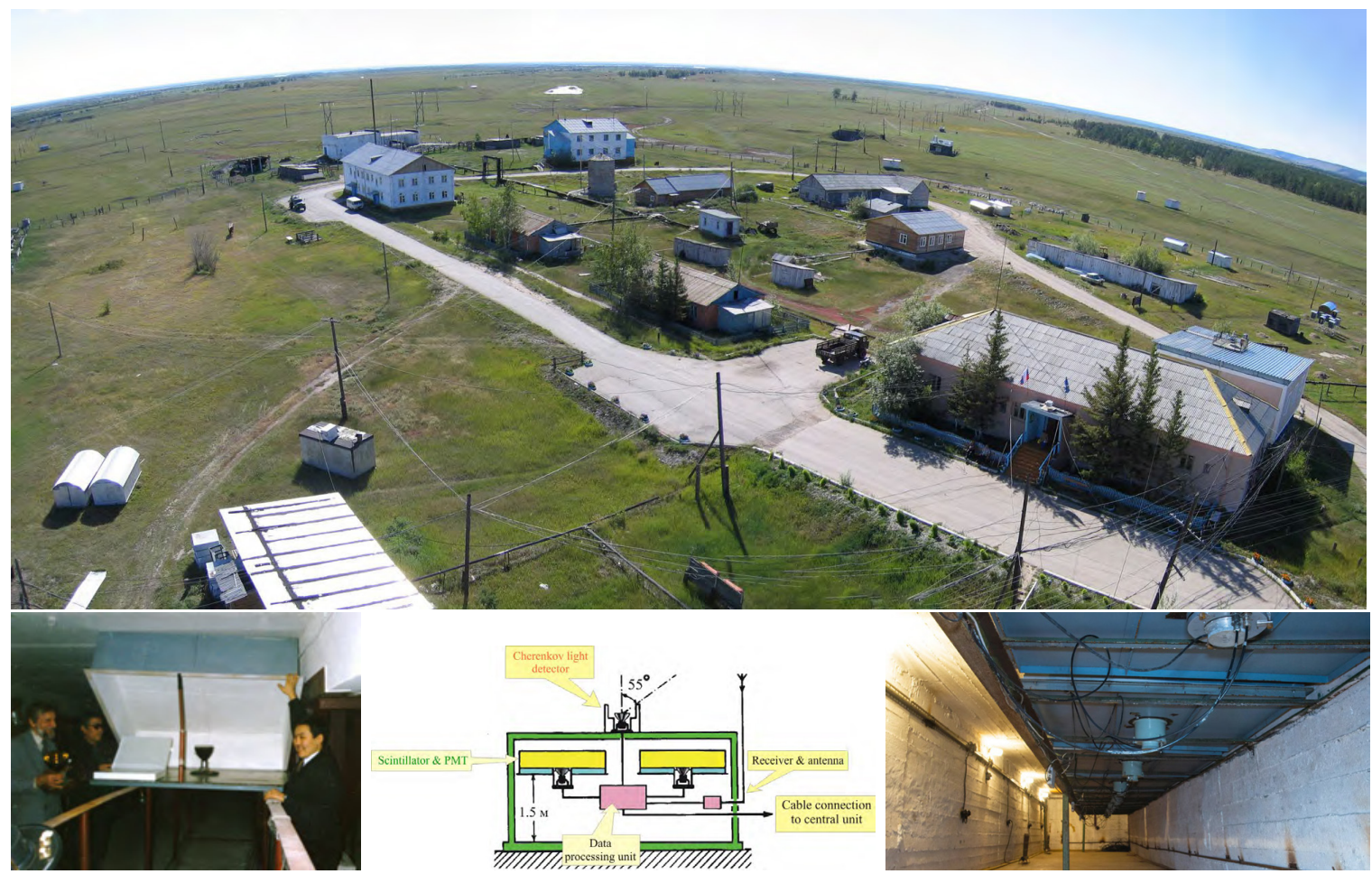

Figure 1. Upper panel: Central part of the Yakutsk array. Lower panel: Left: A box with scintillation counter and PMT. Center: Layout of the detectors in a station. Right: A muon detector.
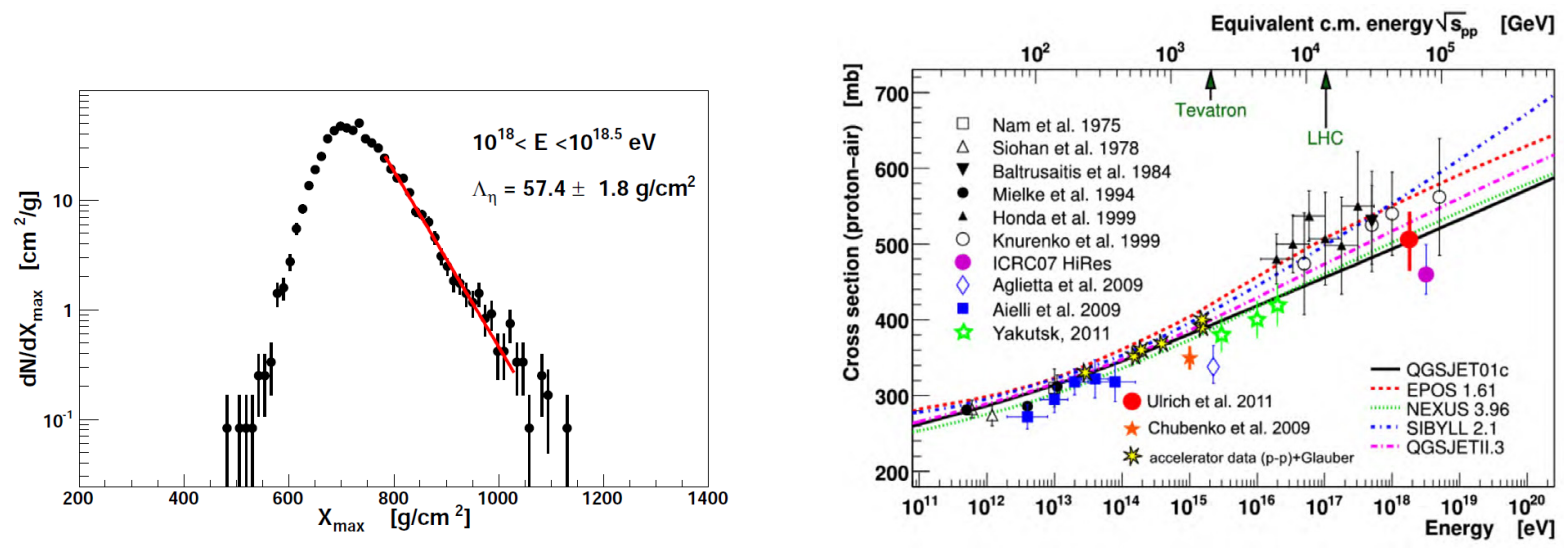

Figure 2. Left: Distribution of $x_{\max }$ in the energy bin. Right: World data on $\sigma_{p}^{a i r}$ estimation in cosmic ray experiments.

imated by the log-normal distribution. As a result, the close connection between the shower absorption rate in the atmosphere and the zenith angle distribution of the rate of EAS events at the observational level is demonstrated by comparing the observed and expected distributions.

The position of the shower maximum ${ }^{1}$ in the atmosphere, $x_{\max }$, is one of the main characteristics of an EAS. Our results [14] are shown in the context of global data from EAS arrays in Fig. 4, which is borrowed from [15]. The dispersion in $x_{\max }$ (right panel of Fig. 4) is illustrative of the mass composition evaluation of CRs as a function of energy, and is compared

\footnotetext{
${ }^{1}$ in the number of particles
}

with the model simulations for primary nuclei shown by the curves.

The radio signal detectors of the Yakutsk array operate in the frequency band $30-35 \mathrm{MHz}$ in coincidence with surface detectors. Ten pairs of crossed dipole antennas (oriented east-west vs. north-south) deployed over an area of $0.35 \mathrm{~km}^{2}$ are used to receive the signals from showers [16]. The data acquisition system includes broadband amplifiers, $100 \mathrm{MHz}$ 8-bit ADCs, and a data processing computer. The radio signal from the shower is selected from the background noise using the trigger signal from the array of charged particle and Cherenkov light detectors. 

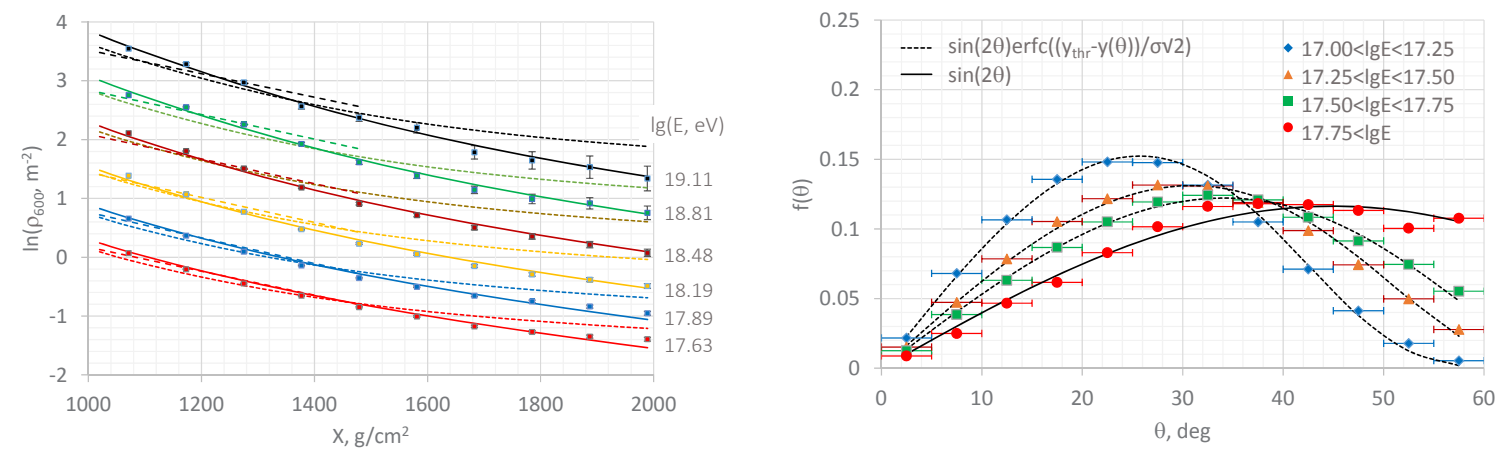

Figure 3. Left: Particle density profile, $S_{600}(x)$, derived using constant intensity cuts. Right: Zenith angle distribution of EAS event rate observed with the Yakutsk array.
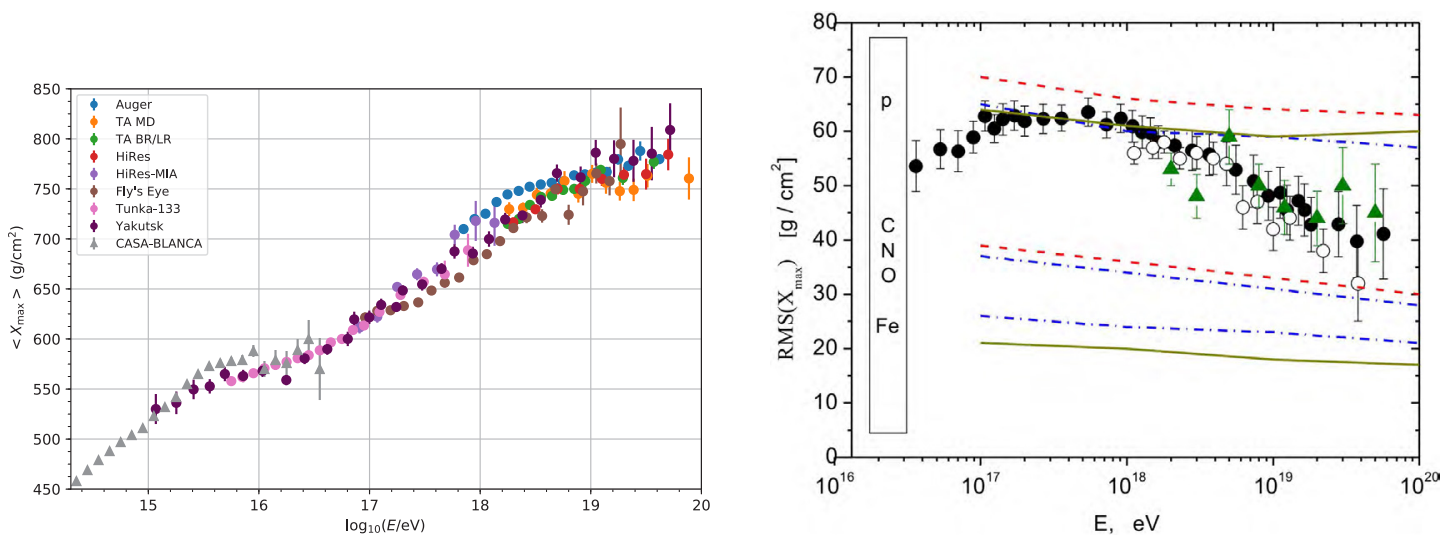

Figure 4. Left: Estimation of $x_{\max }$ in EAS experiments. Right: RMS deviation of $x_{\max }$ measured in experiments and model simulations.
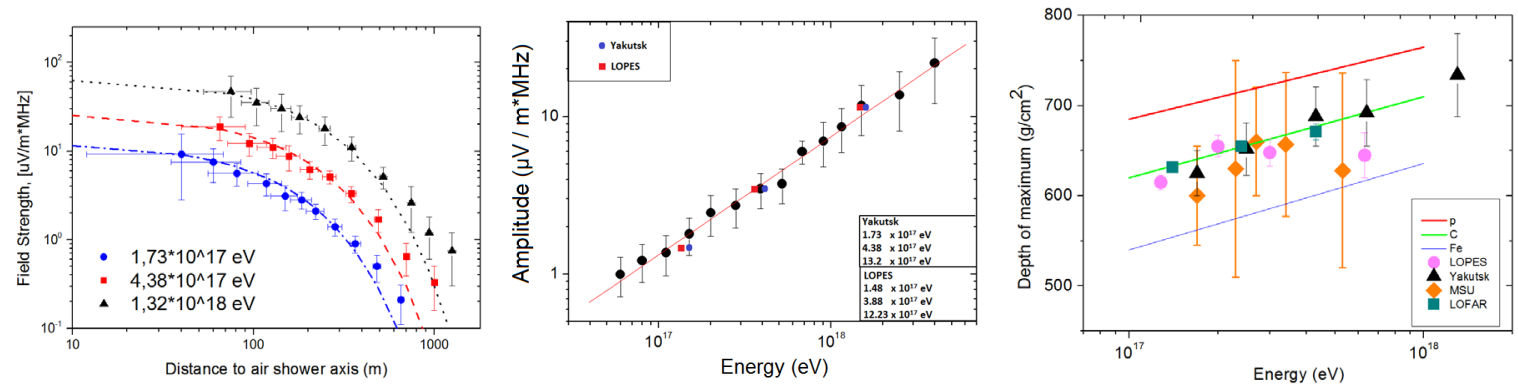

Figure 5. Left: Field strength vs R. Center: Amplitude vs E. Right: $x_{\max }$ reconstruction with radio signal.

The resultant radio signals from the EAS with energy $E>10^{17} \mathrm{eV}$ are attributed to showers with characteristics defined by the main array. Fig. 5 shows the lateral distribution of the radio signal as a function of the shower core distance, the dependence of the amplitude on the primary energy, and $x_{\max }(E)$ estimated using the radio signal.

The results demonstrate the emission of a radio signal by the EAS with energies between $10^{17} \mathrm{eV}$ and more than $10^{19} \mathrm{eV}$. The radio signal is also detected in inclined showers, confirming the geomagnetic mecha- nism of radio wave generation by relativistic particles in showers.

Our results prove that the radio signal from EAS detected at energies above $10^{17} \mathrm{eV}$ can be used to study the physics of air showers, both as an independent method and in conjunction with other methods.

\section{The astrophysics of UHECR}

The energy spectrum of CRs, as measured using the EAS array, essentially depends on the accuracy of the primary energy estimation method. The latest improvement in the algorithm used by the Yakutsk 

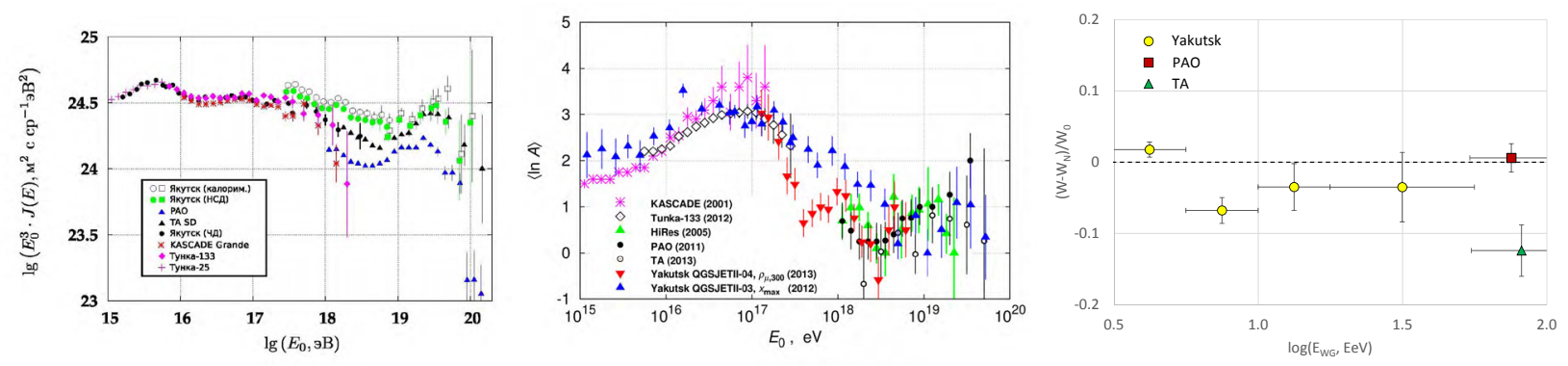

Figure 6. Left: Differential energy spectrum of CRs. Center: Mass composition of CRs estimated from EAS array data. Right: The minimum width of UHECR arrival directions distribution.

array group was reported recently [17]. The resultant energy spectrum combined with data from the Cherenkov detectors [18] is shown in the left panel of Fig. 6, in comparison with the results from other arrays. At the highest energies, there are still some discrepancies between the data; however, the main features of the spectrum, i.e. the knee, ankle and cutoff, appear to be compatible. Moreover, an indication of a second knee at an energy of $\sim 2 \times 10^{17}$ $\mathrm{eV}$ is obtained from the Cherenkov light detectors of the Yakutsk array, and this is presumably formed by a sharp steepening of the spectrum of galactic heavy nuclei [19].

The divergence in the energy spectra of UHECRs measured with different giant arrays is actually congruous with instrumental errors if the energy correction factors are taken into account [20]. These factors, which are individual for each particular array, may be attributed to uncertainties in the nucleus-nucleus interaction models used to connect the observed shower parameters with the primary energy of the EAS.

The main methods for estimation of the mass composition of CRs are based on measurements of the mean value of and fluctuations in $x_{\max }$, and the muon content of showers. The results from the Yakutsk array obtained using these two methods $[21,22]$ are shown in the central panel of Fig. 6, in comparison with available data from other experiments. A conventional conclusion from these various results would be that the mass composition changes from a heavy galactic to a light extragalactic CR component between $10^{17}$ and $10^{18} \mathrm{eV}$. The results are more divergent at the highest energies, due to the reduced number of EAS events detected, and possibly due to differences in model-dependent interpretations.

An event-by-event comparison of the muon density observed in an EAS by the Yakutsk array detectors with that expected from CORSIKA simulations for primary protons and iron, using SIBYLL and EPOS hadronic interaction models, suggests the presence of two distinct hadronic components, 'light' and 'heavy', which is in good agreement with the expected composition in which the light component corresponds to protons and the heavy component to iron-like nuclei. Assuming a two-component (proton and iron) com- position and the EPOS model, the fraction of protons with energies $E>10^{19} \mathrm{eV}$ is found to be $0.52_{-0.20}^{+0.19}$ at $95 \%$ confidence level [23].

The arrival directions of UHECRs have traditionally been analyzed by a variety of methods in order to reveal subtle anisotropy. A simple and promising method has been recently proposed by our group, namely, the minimum width of the circular distribution [24]. The idea is that the isotropic distribution is characterized by the maximum width, while a source of CRs, if present, inevitably reduces this width, depending on the fraction of the total flux: the greater the fraction from a source, the smaller the observed distribution width. The asymptotic limiting value is the angular size of the source.

The minimum width calculated using the Yakutsk array data, and a comparison with the widths derived from the available data from the PAO and TA arrays, are shown in the right panel of Fig. 6. Indeed, the method unambiguously reveals the 'HotSpot' in the data from TA, which is hypothesized to be a source of CRs with $E>5.7 \times 10^{19} \mathrm{eV}[25]$.

EAS events induced by photon primaries can usually be identified by a deficit of muons in comparison with nuclei-initiated showers (Fig. 7, left panel). The estimated cosmological photon flux is within the region of interest for highest-energy astrophysics, tests of extragalactic backgrounds and for searches for new physics.

Although the muon energy density is a better method than the depth of the shower maximum for distinguishing primary photons from hadrons inducing EAS, we also used a multi-component analysis of air showers detected by the Yakutsk array at energies above $10^{18} \mathrm{eV}$ [26] to strengthen the upper limit on the integral flux of primary photons in the CR beam in comparison with previous efforts.

As a result, an upper limit on the integral photon flux is derived in the energy interval $\left(10^{18}-10^{19}\right) \mathrm{eV}$, which is stronger than previous results based on an analysis of data from the Yakutsk array [27]. Both results are compared with data from other giant arrays and model predictions in the right panel of Fig. 7. Experimental data disfavor super-heavy dark matter and topological defect models in top-down scenarios. 

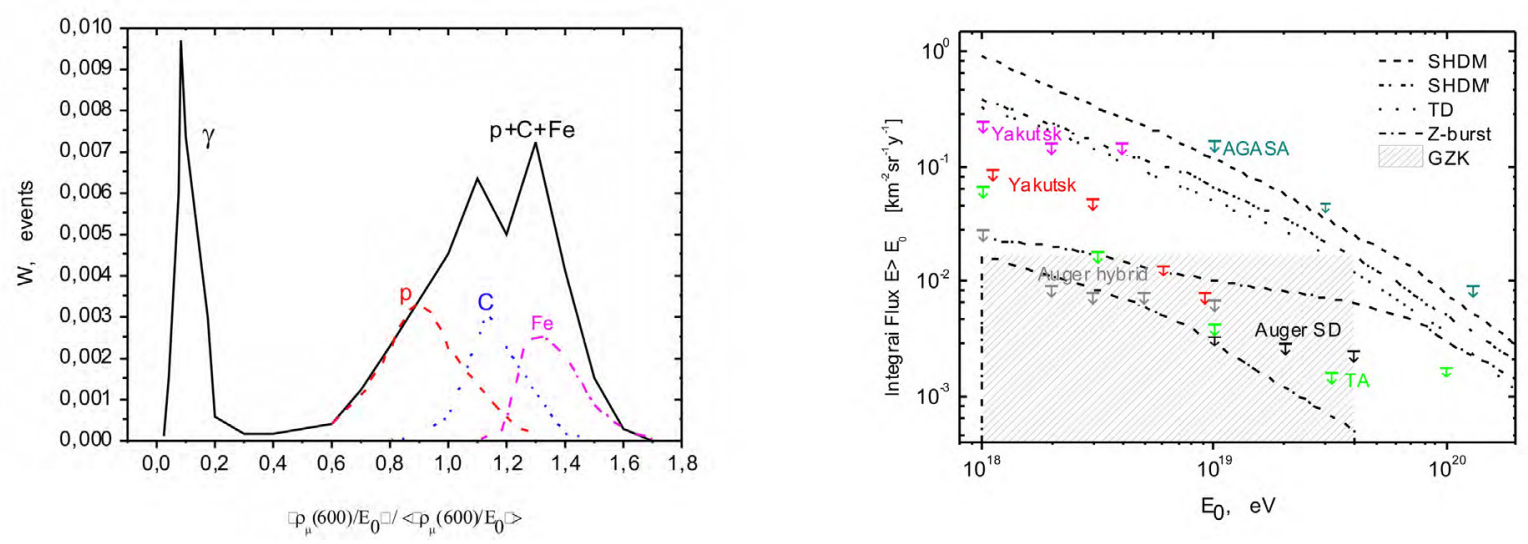

Figure 7. Left: EAS distribution vs muon fraction in the QGSjetII-04 model. Right: Experimental limits on cosmological photon flux and astrophysical scenarios.
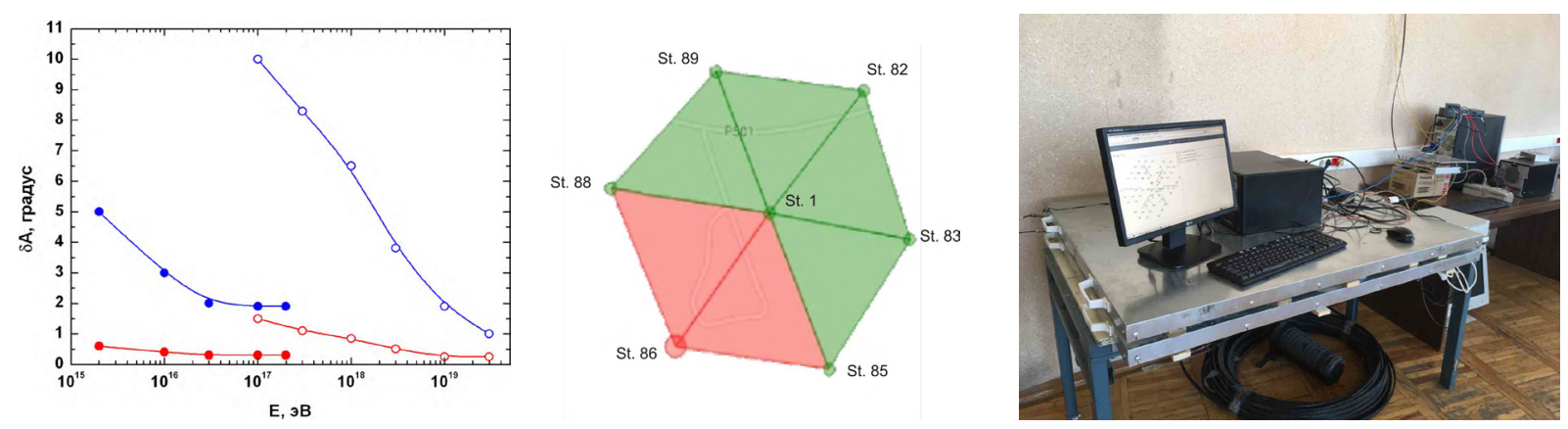

Figure 8. Left: Target (red) and current (blue) angular resolution of the array. Center: The prototype set of modernized stations. Right: A test bed for scintillation counters.

\section{Modernization of the Yakutsk array}

The Yakutsk array needs modernization, and the main goal of this is to improve the accuracy of angular measurements and primary energy estimation. The uncertainty in energy estimation is expected to be reduced with a new set of Cherenkov light and charged particle detectors, while a fast synchronization system will provide the measurement of the signal temporal parameters with improved accuracy.

The new Gigabit Ethernet LAN of the array operates over the optical fiber network connecting all the array detectors. The aim is to provide a synchronization accuracy for the detectors of $\sim 10$ ns with regard to the central time server, as far as to modernize the data acquisition and transfer systems. New scintillation counters are currently being tested on a prototype subset of 11 stations (Fig. 8).

The temporal characteristics of the Cherenkov signal are studied with the wide FOV telescope, which detects showers in coincidence with the surface detectors of the array $[28,29]$. It has been shown that the pulse duration is a function of the shower core distance (Fig. 9). Precise measurements of the time differences between Cherenkov photons arriving at the detectors and the shower core can be used to esti- mate not only the arrival directions of the primaries but also the shower age and $x_{\max }^{C h e r}$.

However, the current accuracy of the Yakutsk array detectors is not sufficient to measure the distance to the shower maximum with reliability. Significant improvements are needed in the timing resolution and synchronization of detectors. The key objective in this context is to improve the synchronization of the array detectors, in order to refine the core location accuracy and the measurement of time differences between the signals at the detectors. When complete, the modernization program of the Yakutsk array will make this type of measurement possible.

\section{Conclusion}

An arbitrary selection of recent results from the Yakutsk array is given in this short review. Although the organizing committee asked preferentially for results related to hadronic interaction models, the majority of the aspects discussed here are related to astroparticle physics. This was a result of the author's intention to present results covering the entire area of research in which the Yakutsk array group is involved. 

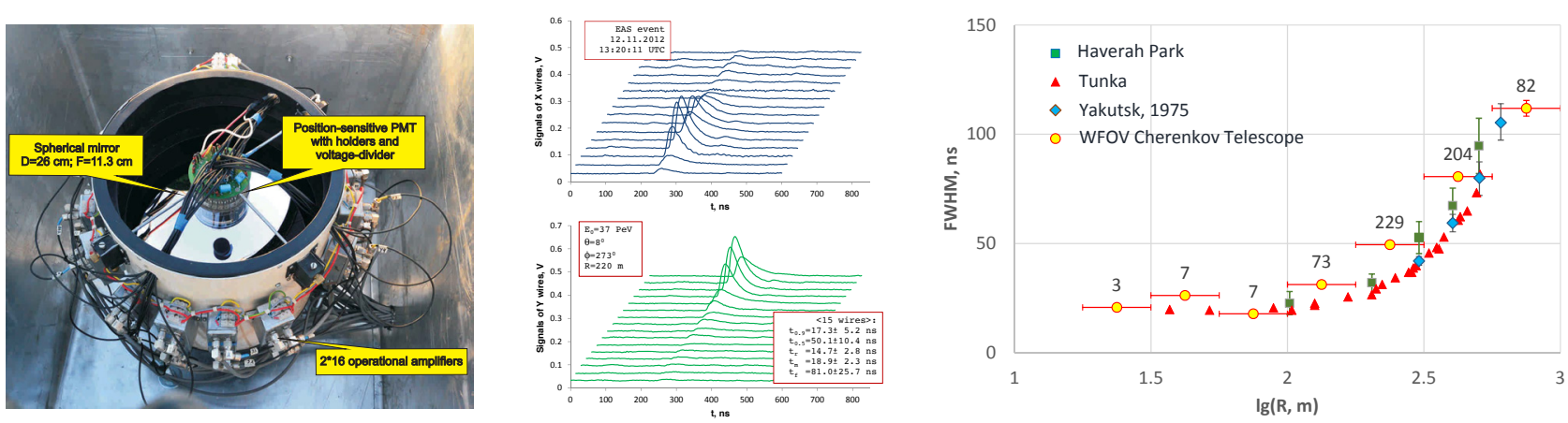

Figure 9. Left: Wide field-of-view Cherenkov telescope. Center: Signals from X- and Y-wires of the multi-anode photomultiplier tube in the focus of the mirror. Right: Pulse duration of the Cherenkov signal vs the shower core distance.

The author would like to thank the organizers of the meeting for the invitation to give a talk and for providing a stimulating atmosphere in the sessions.

This work was partially supported by SB RAS (program II.2/II.16-1), and by RFBR (grant no. 1629-13019).

\section{References}

[1] A.A. Ivanov, S.P. Knurenko, I.Ye. Sleptsov, JETP 104870 (2007)

[2] A.A. Ivanov, S.P. Knurenko, M.I. Pravdin, I.Ye. Sleptsov, Moscow Univ. Phys. Bull. 65292 (2010)

[3] A.A. Ivanov, Nucl. Phys. B (Proc. Suppl.) 190204 (2009); EPJ Web of Conf. 53, 04003 (2013); ApJ 763, 112 (2013)

[4] A.A. Ivanov et al., Astropart. Phys. 621 (2015)

[5] M.I. Pravdin et al., Bull. Russ. Acad. Sci. Phys. 71445 (2007)

[6] A.V. Glushkov et al., Bull. Russ. Acad. Sci. Phys. 55, 717 (1991); Bull. Russ. Acad. Sci. Phys. 57, 91 (1993)

[7] S.P. Knurenko et al., JETP Lett., 83473 (2006); Nucl. Phys. B (Proc. Suppl.) 15192 (2006); Nucl. Phys. B (Proc. Suppl.) 175, 201 (2008); JETP Lett. 86, 621 (2008)

[8] A.A. Ivanov, Dokl. Phys. 52, 423 (2007)

[9] A.A. Ivanov, S.P. Knurenko, I.Ye. Sleptsov, New J Phys. 11, 065008 (2009)

[10] S.P. Knurenko, A.V. Sabourov, EPJ Web of Conf. 53, 07006 (2013)
[11] R. Ulrich et al. (PAO Collaboration), PoS(ICRC2015) 401

[12] J. Alvarez-Muniz et al., Phys. Rev. D 66, 123004 (2002)

[13] A.A. Ivanov, PoS(ICRC2017) 550

[14] I. Petrov et al., PoS(ICRC2017) 331

[15] R.U. Abbasi et al., astro-ph/1801.09784

[16] S.P. Knurenko et al., Nucl. Inst. Meth. A866, 230 (2017)

[17] A.V. Sabourov et al., PoS(ICRC2017) 552

18 S.P. Knurenko, A.V. Sabourov, Izv. RAN ser. fiz. 78, $324(2014)$

[19] S.P. Knurenko et al., EPJ Web of Conf. 99, 04001 (2015)

[20] A.A. Ivanov, JETP Lett. 91, 209 (2010); ApJ 712, 746 (2010)

[21] A.V. Glushkov, A.V. Sabourov, JETP Lett. 98, $655(2013)$

[22] S. Knurenko, I. Petrov, EPJ Web of Conf. 99, 04003 (2015)

[23] A.V. Glushkov et al., JETP Lett. 87, 190 (2008)

[24] A.A. Ivanov, ApJ 804, 122 (2015)

[25] R.U. Abbasi et al., ApJL 790, L21(2014)

[26] S.P. Knurenko, I.S. Petrov, JETP Lett. 107, 709 (2018)

[27] A.V. Glushkov et al., Phys. Rev. D 82041101 (2010)

[28] A.A. Ivanov et al., Nucl. Inst. Meth. A772, 34 (2015)

[29] A.A. Ivanov, L.V. Timofeev, IJMPD, 25, 1650090 (2016) 